\title{
LA CONTRADICCION: ¿VICIO FORMAL O CIFRA DE CONTENIDO?
}

CLAUDIO GUTIÉRREZ

Universidad de Costa Rica

\section{Introducción}

Existen dos modos de ver la contradicción, tanto en el lenguaje ordinario como en los escritos filosóficos. Uno de ellos, que podemos considerar como el concepto lógico o analítico de la contradicción, la presenta como un vicio del razonamiento; su mejor encarnación la encontramos, en la filosofía clásica, en el principio de no contradicción de Aristóteles, según el cual una cosa no puede ser y no ser al mismo tiempo y en el mismo sentido; en la lógica contem. poránea, en el principio de consistencia o congruencia, según el cual la presencia de una contradicción (conjunción de una proposición y su negación) dentro de un sistema lo invalida para todo efecto, pues una falsedad es capaz de producir todas las otras mediante reglas de inferencia; y en el lenguaje ordinario, en todos aquellos usos en que decimos cosas como "los testigos se contradicen" o "tal afirmación es contradicha por los hechos" o "una noción contradictoria en términos". El otro concepto; que podríamos llamar el concepto dialéctico o sintético de la contradicción, presenta a ésta en una luz mucho más favorable; diversos ejemplos de su contexto filosófico son las obras de Kierkegaard o Unamuno, donde la contradicción se toma como síntoma de densidad ontológica; la filosofía existencialista (Gabriel Marcel: "yo soy y no soy mi cuerpo"), que requiere la contradicción como una forma inevitable de expresar lo inefable; el sistema hegeliano, donde la contradicción constituye el método para entender el devenir y al propio 
tiempo la esencia misma de la realidad; y por supuesto el pensamiento marxista, heredero de Hegel, donde la contradicción dialéctica es la herramiento fundamental para la interpretación de las situaciones sociales e históricas. ${ }^{2}$ Ejemplos del lenguaje ordinario serían toda referencia a las contradicciones de la persona o la vida como algo positivo y laudable, o por lo menos interesante; como en el caso de la contradicción analítica, el uso ordinario de "contradicción" en su sentido dialéctico es derivativo de los usos filosóficos mencionados.

Estos dos conceptos o apreciaciones sobre la contradicción naturalmente constituyen, tomados juntos, un ejemplo valedero de contradicción." ¿De cuál de los dos tipos? Se que muchos filósofos de tradicción lógico-positivista no vacilarán en calificarla de contradicción simplemente lógica. Es decir, aceptarán que un conjunto de teorías filosóficas que implican el enunciado "la contradicción es un vicio del razonamiento" y un conjunto de teorías que implican enunciados como "la contradicción es índice de densidad ontológica" podrían ser formalizadas respectivamente como " $p$ " $y$ " $\sim p$ ". De esos dos enunciados, por supuesto, y de acuerdo con el principio de no contradicción, sólo uno de ellos, el primero, será el verdadero; el otro será necesariamente fal. so (de acuerdo con el principio de tercero excluido). Esto si no se da más bien la opinión de que el segundo conjunto de teorías corresponde a una hilación de vocablos, cada uno quizá con sentido, pero que tomados en globo carecen completamente de significación. Por más atractiva que, en su simplicidad y claridad, esta opinión sea, su aceptación con. llevaría el rechazo de tanto material filosófico honrado por

\footnotetext{
1 En lo que sigue supondré que la existencia de dos conceptos de contradicción no constituye un caso de equivocidad. Las razones para esta suposición las presento más adelante en este trabajo.

2 "La contradicción dialéctica (a condición de que sea tal, y no una opo. sición formal o una simple confusión) debe ser tomada como síntoma de la realidad. Sólo es real lo que presenta contradicciones, lo que se presenta como unidad de contradicciones", Henri Lefebvre, Lógica formal y Lógica dialéctica, Siglo XXI, México, D. F., p. 222.
} 
la tradición intelectual y de tantos giros lingüísticos incorporados'en la sabiduría popular que resulta tentador suponer que la otra situación es la verdadera: que la contradicción expuesta constituye una contradicción de tipo dialéctico, indicadora de cierta dimensión profunda de la realidad.

El tema de la contradicción dialéctica está asociado con el aforismo de Hegel "todo lo racional es real y todo lo real es racional". Permítaseme considerar el asunto, sin embargo, únicamente desde uno de estos ángulos, el aspecto epistemológico: todo lo real es racional; lo que existe tiene una dimensión inteligible, y esa dimensión es analizable por separado en la medida en que el hombre le presta vida aparte con su propio pensamiento. Si "la realidad es contradictoria", tal carácter se manifestará entonces forzosamente en nuestro pensamiento sobre la realidad y en el lenguaje con que hablamos sobre la realidad: ahí podremos analizarlo. En cuanto a la primera parte del aforismo, que todo lo racional sea real, y que la contradicción dialéctica que constituye la esencia misma del espíritu deba reflejarse como devenir antagónico en la vida, en el mundo o la naturaleza, permítaseme dejar este aspecto ontológico del enunciado hegeliano fuera de consideración.

\section{Forma y contenido}

El objeto de todo conocimiento es, en último término, algo real y concreto, por más que el conocimiento mismo en la mayor parte de sus expresiones quede formulado en enunciados de carácter general. La finalidad misma del conocimiento, podemos agregar, es también lo real, pues se identifica con la necesidad de orientarnos entre los objetos sensibles cuya colección llamamos corrientemente la vida o el mundo. No obstante, para lograr su fin concreto el conocimiento debe inevitablemente pasar por lo abstracto; para llegar a la sintesis, cuyo resultado será nuestra orientación en el mundo, el conocimiento debe comenzar por ser el análisis en que consiste toda operación separadora de 
factores, elementos o aspectos en la cosa conocida. Creo que en esto me acompañarán sin disentir la mayoría de los lectores: conocer lo concreto es ante todo descomponerlo en lo abstracto; para comprender el texto debemos separarlo del contexto. Si cada texto debe cumplir la función de iluminar y ser iluminado por el contexto, debemos tratar de percibirlo hasta donde sea posible como una unidad en sí mismo, aunque en definitiva su sentido será discernible solamente entendiéndolo en la interacción de todos los textos que integran el contexto. Es obvio que cada separación, cada amputación de contexto, empobrecerá progresivamente al texto resultante, lo vaciará de contenido; el resultado final será una forma, residuo último posible de estas amputaciones que nos dará la identificación de un texto con el mínimo de contexto (quizá solamente indicación de coordenadas espaciales dentro del contexto). Pero no nos dejemos impresionar por estas situaciones límites: el contexto no analizado o el texto aislado de todo contexto, la forma absolutamente vacía de contenido o el contenido desolado de forma. Sabemos que las situaciones límites no son reales sino solamente virtuales; a su vez ellas mismas son únicamente abstracciones, momentos analíticos, instrumentos metódicos, puntos focales por ellos mismos inexistentes o por lo menos supremamente opacos; pero no obstante capaces de verter luz sobre lo comprendido entre ellos como límites, es decir sobre todo lo intermedio, en nuestro caso sobre lo que es forma y contenido al mismo tiempo, en grados de combinación diversos según su cercanía a los puntos focales o extremos.

Así pues, la forma es el objeto de conocimiento en cuanto que separado por el análisis. El contenido, por su parte, es el objeto concebido en sus relaciones innumerables con otros objetos, tal y como lo presenta la sintesis. La forma alude a limitación y a empobrecimiento, pero también a clarificación y estabilización del mundo objetivo; la abstracción o análisis que la produce es acción humana dominadora: di- 
vidimos, aislamos el objeto para mejor controlarlo. La abstracción analítica y separadora es una proyección intelectual de actos prácticos destructivos, como la acción del carpintero que corta y elimina la madera que sobra, o la del herrero que funde con llama o parte con cincel; en su versión extrema y final, el análisis desintegra y acaba, como la herramienta cortante o el compuesto químico corrosivo usado sin la guía y prudencia de la mano diestra y la intuición profesional. Su intención, como la de toda violencia, es el sometimiento de su objeto, su dominio y control; es instrumento de poder. Su carácter mortífero establece un límite para su propia acción: no puede haber un análisis exhaustivo, como no puede haber una violencia absoluta (sobre el esclavo, sobre el enemigo) porque sería una acción que anularía su propio propósito. El esclavo muerto no puede servirnos, y el territorio enemigo quemado no servirá para explotarlo en nuestro provecho. Así, el análisis amputa sentido al texto, pero se detíene ante ese reducto de sentido mínimo que llamamos forma. Por su parte, el contenido alude a vivencia oscura y fluida, pero al propio tiempo a interconexión y riqueza de sentido; la síntesis que nos reconcilia con él es una proyección intelectual de actividades prácticas constructivas: unir por clavo o cemento, armar, soldar, sembrar, cultivar. En su versión extrema o final, sin embargo, la síntesis se pierde en el puro disfrute de una integridad no analizada que sobrecoge y envuelve y en la cual carecemos de dominio. Ese carácter integral de la síntesis también establece un límite para su acción: una reconstrucción total, que borrara toda huella del análisis precedente, recaería en el continuo de lo irracional y nos dejaría sin posibilidad de aprovechamiento de la entidad reconstruida; una síntesis completamente exitosa nos impediría, por desaparición de toda forma preexistente, expresar el objeto de conocimiento y llevar sus conclusiones a la práctica: nos remitiría siempre a algo distinto de lo que estuviéramos considerando, sin darnos oportunidad de comunicar lo sabido o de realizar alguna parte de lo planeado. 
Partes y todo, en un enfoque lingüístico como el que hemos querido adoptar aquí, se interpretan muy bien como texto y contexto. Analizar una pieza lingüística será, pues, textualizarla, separarla en diversos componentes elementales, los menores posibles sin que se sobrepase el límite destructivo del análisis. En esta textualización la globalidad de la pieza será guía y prudencia que permita la aplicación inteligente de la violencia de la abstracción desintegradora. Sintetizar será por su parte reconstruir el contexto, contextualizar la pieza lingüística textualizada previamente. Esta acción reconstructora se llevará a cabo, naturalmente, por etapas, pasando de textos elementales a textos más amplios, tendiendo al contexto más amplio posible. Conocer, según esto, será progresar indefinidamente en la puesta en contexto de los objetos previamente definidos en su entidad elemental por la textualización o formalización. Contexto y formalismo vendrán así a corresponder a dos dimensiones lingüísticas fundamentales: la semántica y la sintática. Forma y contenido son inseparables, no podemos ni siquiera concebir uno de ellos sin el otro. Tampoco podemos aislar sentido contextual y formalismo simbólico: el uno alude al otro y cada uno, de manera residual o germinal, está presente en el otro. La sintaxis es incomprensible sin referencia a un contexto, aunque ese contexto sea reducido, inmediato y manejable de conformidad con reglas claras y rigurosas. Lo sintáctico es el contexto mínimo, en que unos signos se definen como comportamiento regular con respecto a otros signos definidos similar y recíprocamente. Por su parte, lo semántico es el contexto expansivo; se refiere al sentido de los signos, va más allá del contexto formal y por su relación con todo el universo es como una flecha que señalara al infinito. Contexto ilimitado o progresivamente expandible, he ahí lo que caracteriza a la dimensión semántica del simbolismo: el signo remite fuera de sí, en círculos concéntricos de significación cada vez más amplios cual campo de fuerza. Lo sintáctico es fundamentalmente materia de cálculo; 
apela a lo que los filósofos han llamado entendimiento. Lo semántico es fundamentalmente materia de reflexión; apela a lo que los filósofos tradicionalmente llaman razón.

Todo pensamiento, inevitablemente, es en parte sintáctico, porque tiene estructura además de sentido. De lo contrario, si la estructura faltara o hubiera quedado borrada por la síntesis, la reflexión, búsqueda de sentido y descubrimiento de conexión, avasallaría con su carácter indefinidamente expansivo al mismo pensamiento; lo obnubilaría, le impediría manifestarse verbalmente y transferirse a la práctica. ¿Cómo decir lo que pensamos si nuestra reflexión nos remite constantemente a otra cosa, sin dejar de poner todo símbolo en un contexto cada vez más amplio? ¿cómo realizar una acción cualquiera si no sabemos todavía hacia adónde vamos o por qué debemos movernos? Así pues, el pensamiento, aun el más reflexivo, debe ponerse límites a sí mismo, para canalizarse hacia la expresión y la acción. El pensamiento se construye diques a sí mismo, que son la estructura lógica o formal de proposiciones y razonamientos. El pensamiento se determina o define, no queda indefinidamente flotando en la indiferencia eterna y en la duda creadora. Pero por otra parte, tampoco puede ser el pensamiento totalmente formal o sintáctico; toda forma es en cierto modo algún contenido, no se independiza completamente del contenido. Formalizar es suprimir contenido, pero no todo contenido: es sustituir un contenido por otro que en algún sentido y para cierto propósito se considera más simple. Si a la forma le quitamos un contenido por ese mismo hecho le prestamos un contenido distinto, pues toda formalización significa en último término la construcción práctica de un modelo que es también contenido, aunque presuntamente diferente. Esto lo sabe muy bien el programador electrónico reflexivo: cuando se le pide independizar su algoritmo de un lenguaje cibernético determinado y producir la "lógica" del programa en sí misma, no tiene más remedio que traducir su algoritmo a otro lenguaje, más cerca del len- 
guaje ordinario, o bien producir un modelo pictórico, el "diagrama de flujo", que es también por supuesto un lenguaje con su propia dimensión semántica o contenido. La pura estrucutra, sin ningún sustrato material que la exprese o contenga, simplemente no existe, no puede existir. Las relaciones matemáticas más abstractas, siempre las encontraremos encarnadas en una representación u otra: serán manchas de tinta en un papel, o anillos magnetizados en las entrañas de una computadora, o configuración de impulsos eléctricos y neuronas en el cerebro humano. Por lo demás, la supresión de contenido, quiera decir esto lo que quiera decir, es sólo transitoria e instrumental, un momento en el método de análisis y síntesis que hemos explicado: se aisla únicamente para reintegrar, nos sumimos en lo abstracto únicamente con el propósito de regresar, más tarde o más temprano, a lo concreto.

Síntesis y análisis son inseparables; además, se interpenetran recíprocamente. Su funcionamiento sólo puede entenderse, por lo menos desde una perspectiva naturalista, como la interacción de postulados hipotéticos extremos: postulamos las. últimas partes que den forma a una síntesis y postulamos un todo final que de sentido al análisis, pero partes y todos sólo cobran forma y sentido progresivamente, sin que nunca lleguen a tenerlos independientemente del proceso que nos lleva a ellos. Los postulados iniciales los justificará el proceso subsecuente; los finales, el proceso antecedente; y el conjunto total de postulados y proceso lo justificará sólo la praxis integral del sujeto involucrado. En el fondo, los postulados iniciales sólo pueden tener su origen en actitudes prerracionales, puesto que el proceso de crítica y construcción racional no ha comenzado. Lo irracional aparece así como inerradicable, aunque progresivamente menor en la composición orgánica del conocimiento: permanece residualmente en toda síntesis progresiva como un testimonio histórico del carácter eminentemente hipotético y asimptótico de todo conocimiento. En palabras 
de Henri Lefebvre, avanzamos de la ignorancia al conocimiento; en este avance, la síntesis guía al análisis y el análisis fundamenta a la síntesis, pero nunca logramos un análisis completo (no basado en una hipótesis sobre el todo) ni una síntesis total (no basada en una hipótesis sobre las partes). De ahí que, en suma, debamos mirar con desconfianza un análisis pretendidamente exhaustivo y una síntesis pretendidamente reconstructora total; análisis y síntesis tienen naturalezas focales, tan inexistentes en sí misma como la pura forma o el puro contenido. Su verdad es, más que existencia separada, interacción: conocer es moverse del análisis a la síntesis, en rítmicas pulsaciones de ida y vuelta, sin que ninguna de las dos direcciones desaparezca completamente $;^{3}$ ir del todo a las partes, sin que el todo deje de iluminar la actividad del análisis, y de las partes al todo, sin que las partes dejen de hacer inteligible al resultado de la síntesis.

\section{La esencia}

Conocer es asimilar esencias. La depuración de la filosofía de toda forma de idealismo o metfísica, creo yo, no debe lograrse al precio de suprimir el uso de este término del discurso filosófico; tal supresión sólo llevaría al angostamiento del lenguaje y a una filosofía empírica engañosa. Lo cierto es que hoy, tanto como en la época de Aristóteles, no puede haber ciencia de lo no esencial, aunque hallamos progresado muchísimo en la interpretación de lo que es o no esencial. El progreso fundamental ha consistido en que ahora entendemos que no hay nada que no pueda llegar a ser esencial desde un punto de vista determinado, dentro de algún tipo de contexto. Dicho de otra manera, hemos comprendido que al esencia es siempre rẹlativa al contexto, que lo que es esencial desde un punto de vista deja de serlo

3 Confróntese la exposición sobre el "va-et-vient" del método heuristico en J. P. Sartre, "Question de Méthode", Critique de la raison dialectique, Gallimard, París, 1960, pp. 87 y ss. 
desde otro, y viceversa. Es decir, que no hay esencias o verdades eternas, independientes de contexto. El rechazo del idealismo o de la metafísica esencialista debe consistir en rechazar esa pretendida independencia de la esencia, no en rechazar el concepto mismo. El concepto "esencia" sigue siendo necesario, aunque deba recibir una nueva interpretación. Suprimirlo del lenguaje filosófico exigirá tener que inventar otro nuevo término, que tuviera el viejo sentido de "esencia" aunque enriquecido y renovado. Los términos, como las personas, cambian su contenido con el pasar del tiempo y el intercambio con un cambiante contexto lingüístico o social. Pero no porque nos desarrollemos emotiva o intelectualmente debemos cambiar de nombre o de cédula de identidad. Permanecemos los mismos bajo los antiguos símbolos, pero redefinidos en nuestra significación por lo que podríamos llamar evolución contextual. El concepto "esencia" es a su vez una esencia; no eterna, sino histórica. Tratemos de aclarar cómo interpretarla en su nuevo contexto, en su contexto de hoy, porque tampoco ella, al igual que las otras esencias, es independiente de contexto.

Conocer es definir o explicar causalmente. Ahora bien, definir es mostrar esencias; explicar causalmente es trabajar con esencias. El empirista más exigente se siente autorizado a decir que lo de más acá o lo de más allá es esto o aquello; el correlato del "es", de la cópula, constituye lo que por múltiples razones conviene seguir denominando esencia. $Y$ en cuanto a las generalizaciones explicatorias de las ciencias, siempre tienen que ser esenciales, relativas a la esencia, y no ser de carácter fortuito como la generalización accidental "todas las monedas en mi bolsillo son de diez céntimos". La lógica misma, la más formal y menos metafísica de las disciplinas filosóficas, tiene que hacer uso de la esencia y suponerla; de lo contrario no podría trabajar con términos o predicados, que no son sino una versión formalizada (côn mínimo contenido) de las esencias. De otra manera, la lógica supone la existencia de tipos reales 
en que se clasifican los objetos; no podría haber lógica sin cierta presuposición de regularidad en la naturaleza que agrupe a los objetos en clases. La lógica en sentido pleno es lógica de clases. $Y$ si la naturaleza es regular y los objetos se dejan clasificar entonces tenemos derecho a decir que hay esencias. Lo que no es equivalente a aceptar que las esencias o clases sean eternas o independientes de contexto. No estamos afirmando que el mundo es regular y los objetos se dejan de clasificar porque hay esencias; tal vez suceda precisamente a la inversa. Pero si hay regularidad, necesitamos de las esencias para hablar de ella, tenemos definitivamente que contar con las esencias.

Según lo analizamos en la sección anterior, todo lo real o concreto es contenido, implicación de contexto, interconexión de objetos, interacción de acontecimientos. En la era de la teoría de la relatividad y de la reducción práctica de la materia a la energía, en la era del electromagnetismo y los campos de fuerza, tenemos que decir que todo lo que existe es, como soñaba Leibniz, un haz de relaciones. El universo puede concebirse como una inmensa red en que cada objeto se define por el entrecruzarse de diversos hilos que remiten a otros objetos. Los tipos o esencias son nodos especialmente densos en esa red de interconexión en que consiste lo real. La posibilidad del conocimiento supone que somos capaces de percibir esos nodos, de distinguirlos sobre otras conexiones más tenues. Todo conocimiento supone que podemos separar, por lo menos intelectualmente, lo esencial de lo accidental, las conexiones más ricas de las menos ricas. Pero lo esencial y lo accidental forman un continuo; no hay distinción de naturaleza entre las conexiones fuertes y débiles, entre las ricas y las pobres. El gradualismo es lo que impera, con la posibilidad de concentraciones cuánticas aquí y allá que nos permiten hablar de líneas divisorias, definiciones y esencias. "La naturaleza no da saltos" es una verdad fundamental, siempre que tomemos en cuenta el contexto para discernir lo esencial de 
lo no esencial; en un contexto más amplio, la graduación puede dejar de percibirse e interpretares como hiato o salto entre dos esencias. La esencia no sólo es interpretarse como hiato o salto entre dos esencias. La esencia no sólo es contextual sino que admite grados. Hay lo más y lo menos esencial. Una ley causal, una definición, un enunciado de esencia es un corte intelectual en una parte densa en la red de los hilos que interconectan todos los fenómenos. Aunque inteligibles por sí mismos, nos remiten al más allá que configura el contexto total de las apariencias

Todo lo real es interconexión; el conocimiento es real; así pues, el conocimiento es también interconexión. Pero el conocimiento tiene por objeto algo real, o sea, una interconexión. De ahí que el conocimiento sea una interconexión triple. Para conocer interactuamos con la conexión que es el objeto y en consecuencia también con el contexto de ese objeto. No se da nunca la pareja sujeto-objeto; se da más bin el triángulo sujeto-objeto-contexto. Básicamente, y para una perspectiva naturalista, la relación sujeto-realidad es de un carácter idéntico a la relación objeto-contexto: ambas son relaciones materiales, de contenido; el conocimiento no es quehacer puramente formal o ideal. La interacción de la conciencia con la realidad es una actividad, no una forma; es un contenido material. Para conocer la esencia debemos interactuar prácticamente con ella; trabarnos en lucha con ella, la lucha cuyos momentos son el análisis y la síntesis. Al contexto real de la cosa agregamos el contexto teórico del conocimiento y ambos contextos se afectan recíprocamente. El descubrimiento de la esencia, como toda otra creación o transformación de mundo, es y sólo puede ser resultado de un trabajo. Por lo tanto, y naturalmente, ha de ser gradual e histórico, ese avance de la ignorancia a la ciencia a que me referí anteriormente. Avanzamos de la ignorancia a la sabiduría a través del doble trabajo de análisis y síntesis que comenté en la sección anterior, dos maneras de actualizar la interacción entre conciencia y realidad, mente y esencia. Analizar o computar es manejar símbolos, 
otras tantas fichas en el tablero de un juego formal. Sintetizar o reflexionar consiste en dejarse llevar por el contexto, tal vez no expresamente presente en la formalización pero siempre vagamente intuido en el pensamiento más estructurado. El momento formal del conocimiento implica un forzar el contexto fuera de la interconexión triple para pretender metódicamente una relación doble entre la mente y el objeto. Pero el contexto eliminado reacciona filtrándose en el resultado del análisis y se manifiesta ahí como inadecuación en la estructura. La reflexión percibe en la forma independizada de contexto anomalías que son clave de redefinición, inadecuaciones que apuntan a la necesidad de restituir o ampliar el contexto. Esas claves, frecuentemente, constituyen contradicciones, es decir, resquebrajaduras en la estructura formal. Tales contradicciones nos obligarán a la postre a modificar nuestros contextos teóricos para adecuarlos a contextos percibidos cada vez más amplios en la naturaleza y en la sociedad.

Creo que en este sentido, pero únicamente en este sentido, es que la contradicción es signo, síntoma o cifra de la riqueza de lo real. Una contradicción en sentido lógico propio, una contradicción analítica, es descubierta por la conciencia, en función formal o computadora, dentro de un determinado contexto o estructura; dicho descubrimiento ocurre normalmente sólo en zonas periféricas no bien exploradas de la ciencia respectiva, o por el contrario en zonas tan centrales o fundamentales que corrientemente se toman por descontadas y no se someten a intenso escrutinio. Pero cuando la aparición de considerables perturbaciones teóricas que no pueden descontarse como errores experimentales o anomalías reservadas para estudio posterior llaman la atención de los científicos sobre esas zonas, la contradicción no puede ya disimularse." Cabe hacer notar que en una situación crítica tal, la reacción posible de la concien-

4 Confróntese Thomas Kuhn, The Structure of Scientific Revolutions, Chicago: the University of Chicago Press, 1964, pp. 82-83. 
cia no es únicamente el rechazo del paradigma así infectado, o el desaliento y abandono del campo de investigación: es siempre posible preferir por razones pragmáticas mantener la teoría en la ignorancia del contexto más amplio y salvaguardar su congruencia al precio de una menor amplitud de contenido. Se sacrifica así la completitud a la consistencia, recurso optativo para el investigador pero que puede ser obligatorio en algunos casos. ${ }^{5}$ Determinadas definiciones, operativas dentro de un contexto y sólo dentro de él, se transforman en contradicciones al ser ampliado el contexto. La ampliación del contexto justifica, y en realidad exige, el cambio de la definición. Mientras no se dé ese cambio, pero ya dentro del contexto expansivo, la necesidad de redefinir reviste la irritante y llamativa figura de la contradicción. En ese sentido es claramente señuelo, flecha indicadora, de ese contenido real, expresable en nuevos contextos posibles que el contexto estrecho no habría sospechado. La contradicción llega entonces a ser, en vez de vicio formal, índice o cifra de contenido, ventana de la esencia. Encontrar contradicciones es encontrar esencias pues la contradicción obliga a una ampliación de contexto que dota de un contenido más rico a las antiguas definiciones. Habrá contradicciones severas o leves, ligeras o importantes, como definiciones más o menos ricas, esencias más o menos densas. La contradicción admite grados, como la esencia. La "contradicción principal" o sea la más contradictoria, la que exige más severas reformulaciones de contexto para ser removida, será la esencia de la situación en el sentido más propio de la palabra.

Nuestro avance gradual hacia el conocimiento implica trabajo en el doble sentido de imponernos a la realidad,

5 Aludo aquí a la demostrable incompletitud de las matemáticas. Ver Kurt Gödel, "Uber formal unentscheidbare Sätze der Principia Mathematica und verwandter Systeme", Monatshefte für Mathematik und Physik, vol. 38 (1931).

B Confróntese el tratamiento de "la contradicción principal" en Mao Tsetung, Las contradicciones, Grijalbo, México, D. F., 1969, pp. 51 y ss. 
por el análisis, y dejarnos avasallar por la realidad, en la síntesis. Nuestra actividad positiva consiste en poner diques a la realidad, para hacerla manejable (acción que será negativa para la realidad, por ser separadora e in extremis destructora); pero esos diques deben ser a su vez removidos, destruidos, para dar lugar a nuevas perspectivas totalizadoras, nuevas síntesis de lo concreto. Esa será actividad negativa, la negación (por nuestra parte) de la negación (que habíamos puesto antes en la realidad), supresión de una separación (acción que será positiva para la realidad por su carácter restaurador del fluir material e histórico de las cosas).? El pensamiento se construye diques para sí mismo pero luego rompe esos diques para seguir su marcha. Tal afirmación no es novedosa: equivale a declarar que el pensamiento es dialéctico. Quisiera agregar que esa marcha va de la definición o esencia (en un contexto) a la definición o esencia (en contexto más amplio) pasando a través de la contradicción y la superación de esa contradicción por un proceso redefinitorio.

\section{La contradicción y su superación}

El pensamiento debe permanecer congruente consigo mismo a riesgo de destruirse como tal pensamiento. Un pensamiento incongruente es un pensamiento inútil, incapaz de mediar entre una persona y otra, entre mi yo anterior y mi yo inmediato siguiente, entre una situación incómoda y su planteamiento como problema, entre un planteamiento y su solución. No es eficaz como instrumento de análisis ni de síntesis ni como vehículo de expresión de mi relación con el mundo o vínculo de comunicación entre los hombres. No puede cumplir su función enriquecedora- de la práctica a

7 Queda aquí planteado un tema de reconciliación entre las filosofías analítica y dialéctica: el doble concepto de "negación de la negación", que para unos resulta en una simple afirmación y para otros en una afirmación enriquecida dialécticamente. Creo que la reconciliación puede alcanzarse por un camino paralelo al recorrido aquí para reconciliar los dos conceptos de contradicción. 
través del cómputo y la reflexión ni su función unificadora de conciencias y voluntades. Que el pensamiento debe ser congruente es lo que expresaba la lógica tradicional con el famoso principio de no contradicción y lo que expresa la lógica moderna con el criterio de consistencia o congruencia: no es posible que algo sea y no sea al mismo tiempo y en el mismo sentido; un sistema de proposiciones no debe contener entre sus teoremas tanto " $p$ " como "no p". No puede haber articulación posible de la actividad intelectual sin respeto a este principio; él constituye la base de toda forma, de todo análisis, de toda abstracción, de toda hilación. Durante un discurso, un razonamiento, una investigación o una discusión cada término debe conservar su sentido, establecido por una definición previa: de lo contrario no habrá validez de inferencia, ni corrección de formalización, ni legitimidad de método, ni honestidad de conversación. La congruencia, vista así, puede ser tomada como otra manera de mencionar la actividad intelectual misma. En realidad, no es ni siquiera concebible una actividad intelectual que no respetara este principio: el entendimiento humano se agota, la imaginación paradigmática naufraga, si tratamos de concebir un mundo, una situación, un contexto en que el principio de congruencia no estuviere vigente. $\mathrm{Y}$ sin embargo, se nos dice desde posiciones como el taoísmo (con sus oposiciones de yang y yin), la dialéctica de Hegel y Marx o el existencialismo contemporáneo que la contradicción es síntoma de lo concreto y de profundidad ontológica. ¿Estaremos aquí en presencia de un simple equívoco? ¿será una de las dos posiciones un total error? ¿o será ésta una situación de pensamiento analógico, y podremos decir que la contradicción, como el ser de Aristóteles, se dice de muchas maneras?

Consideremos ante todo la posibilidad de que estemos en presencia de un equívoco, es decir, de que cuando alguien reniega de la contradicción como de un vicio del razonamiento esté usando el término "contradicción" en un sentido totalmente distinto del sentido de "contradicción" del que 
usa ese término para recomendar la contradicción como cifra de contenido esencial. Si en el primer caso el término equívoco significaría incongruencia, absurdo o sinsentido lógico o lingüístico, en el segundo significaría más bien algo totalmente distinto, a saber, conflicto práctico, oposición de hecho, enfrentamiento de fuerzas de signo o dirección contrarios. El problema con esta interpretación de la situación es que con seguridad no será aceptada por los que sostienen que la contradicción es revelación o marca de esencia; no aceptarán que lo que quieren decir con su uso del término sea conflicto de hecho, porque están convencidos de que sus contradiciones son materia de lógica profunda y no simplemente de oposición superficial. Su visión del mundo descansa en la pretetensión de que de alguna manera es posible "superar" el principio de no contradicción que según ellos es aplicable solamente a situaciones estáticas pero no al devenir de lo real. No acertaríamos, pues, si tomáramos su uso del término como una metáfora o hipérbole que sólo dotara de fuerza dramática a su convicción de que el mundo es campo de batalla de toda clase de tensiones opuestas. Ellos insistirán más bien en que el ser es y no es, en que " $A$ " es simultáneamente "no $A$ " o en que válidamente puede afirmar dentro de un sistema al propio tiempo " $p$ " $y$ "no p". Por otra parte, es posible señalar en la historia de las ciencias casos de proposiciones contrapuestas que sin embargo en un momento dado, y mientras la contradicción no fue removida por una reestructuración contextual o paradigmática, hubieron de mantenerse ambas dentro de un sistema científico (el éter existe y no existe; la luz es onda y no es onda). Finalmente, las más interesantes oposiciones que señala el análisis dialéctico, como por ejemplo entre amo y esclavo, capitalista y asalariado, implican que cada uno de estos términos contradice al otro no de manera externa, en el sentido de tener intereses opuestos; sino también desde dentro: el esclavo existe por el amo y el amo por el esclavo, en el sentido de que cada uno lleva al otro en su conciencia y no podría ser lo 
que es sin la esencia del otro. $\mathrm{El}$ intento de presentar estas oposiciones dialécticas en forma de relaciones puramente externas, que no impliquen contradicción en sentido lógico, parecen condenadas al fracaso; por lo menos exigirían un cambio tan grande de los paradigmas dentro de los cuales tienen sentido que los llevaría más allá de todo reconocimiento posible. Este tipo de contradicciones es por supuesto diferente de las contradicciones que surgen dentro de un paradigma científico en momentos de crisis; son más bien hipótesis explicativas perfectamente asentadas dentro de paradigmas establecidos. Creo sin embargo que su naturaleza puede asimilarse al género de las contradicciones dialécticas antes mencionadas, pues su valor explicativo va ligado siempre a una definición que entra en crisis, la definición de la propia identidad de una persona o clase social, por ejemplo; y es esa relación con la definición lo que me parece crucial en el análisis de la contradicción dialéctica.

Como consecuencia de lo dicho en el párrafo anterior, es más prometedor considerar el doble uso del término "contradicción" como un caso de analogía y no de equivocidad. En consecuencia, debemos esperar que los dos usos tengan un núcleo de significación común, mientras que uno por lo menos de ellos posea un excedente de significado que lo diferencie del otro. Por la misma razón de que el uso analítico de "contradicción" es más claro que el uso dialéctico, fundamentalmente opaco como hemos visto, es conveniente postular que el significado común sea equivalente a la totalidad del significado del uso analítico; esto es, que el uso dialéctico de "contradicción" califica a una situación de contradictoria en el sentido analítico y además nos dice algo importante por medio de un exceso de significación sobre esa misma situación, o sobre los moldes teóricos que sirven para analizarla; a saber, que la contradicción analítica podría resolverse si tuviésemos éxito en una conveniente redefinición del contexto que dio origen a la situación contradictoria. Planteemos la perspectiva de una manera todavía más completa. Los dos usos de "con- 
tradicción", al oponerse recíprocamente, exigen un trabajo de reconciliación lingüística. La reconciliación puede encontrarse por una de tres distintas avenidas: o bien el término es equívoco, y no hay verdadero choque entre los usos analítico y dialéctico; o bien el término es univoco, y uno de los dos bandos está en un craso error por no aplicar correctamente el término; o bien el término es análogo y entonces sí tenemos un auténtico problema de análisis de significado, que podemos resolver si presentamos una hipótesis plausible de cómo la significación principal del término se flexiona o declina para dar lugar a los distintos usos.

Volvamos ahora al planteamiento original de nuestro problema. Existen dos maneras de ver la contradicción en el lenguaje ordinario y en las disciplinas filosóficas: para el punto de vista analítico, la contradicción es un vicio del razonamiento y tiene carácter predominantemente negativo. ${ }^{8}$ Para la reflexión dialéctica la contradicción aparece grávida de significado y puede entenderse como cifra y expresión de la inexhaustibilidad de lo real. Estos dos punpuntos de vista sobre la contradicción constituyen ellos mismos un ejemplo de contradicción. Lo interesante del caso es que sin un reconocimiento de la vigencia, en algún sentido y medida, del principio de no contradicción este problema de la contradicción ni siquiera podría plantearse. No se podría alegar que la contradicción es síntoma de lo concreto e indicador de riqueza esencial si la contradicción no fuera efectiva y formalmente demostrable: una seudocontardicción no cumpliría el propósito de sugerir la inagotabilidad de lo real. Por otra parte, si la contradicción es efectiva, no es menos cierto que se impone un esfuerzo por

\footnotetext{
8 La inadecuación del contexto para abarcar la realidad tiene dos modos de manifestarse: o como choque de dos proposiciones contradictorias o como insuficiencia del paradigma para dar razón de algún sector importante de la realidad, como su agotamiento frente a la realidad (Cfr. mi "La Abstracción y los Límites de la Imaginación Paradigmática", Crítica, Vol. 2, No. 5). No es sólo el principio de congruencia sino también el de completitud lo que entra en juego aquí.
} 
resolverla o superarla, pues el apetito de congruencia expresado en el principio de no contradicción establece que toda contradicción es intolerable y debe ser eliminada para continuar expresándonos con sentido. Esta exigencia, este apetito por la remoción de la contradicción, nos pone en el camino de lo que creo que es la solución a nuestro problema. En efecto, es esencial a toda contradicción su carácter de intolerable. La contradicción es insoportable y pone al pensamiento a moverse para superarla. La contradicción resulta motor del pensamiento, precisamente por su carácter de intolerable. Lo cual pareciera llevarnos a una nueva contradicción: el pensamiento debe ser congruente, a riesgo de destruirse como tal pensamiento; pero la contradicción da vida al pensamiento, puesto que lo hace moverse. Esta aporía, sin embargo, se disuelve al percatarnos de que la incongruencia ha de ser percibida y denunciada como tal, y por eso es que mueve al pensamiento. No es entonces la contradicción simplemente lo que mata al pensamiento, sino la contradicción ciega sobre sí misma, la contradicción irreflexiva. La contradicción reflexiva, por su parte, no sólo no es mortal sino que incluso parece ser inevitable. Todo pensamiento desemboca en contradicciones, en la medida en que termina en una síntesis y no hay síntesis que abarque para el pensar la totalidad absoluta de lo real. El exceso, el plus de realidad que va más allá de la conceptualización lograda, se hace presente dentro de la articulación del pensamiento como exclusión ${ }^{\vartheta}$ o como contradicción. El perfilar una forma implica un corte y un ocultamiento, y esa privación de contexto, al ser examinada directamente, se revela frecuentemente como contradicción o, si se quiere, como insuficienciá de una definición que clama por un contexto más amplio. La contradicción deja de ser el absurdo si se entiende como un llamado del lenguaje a reformular el universo de discurso.

9 Uso aquí el término "exclusión" como paralelo a "contradicción" para calificar los dos tipos más generales de perturbación radical de un esquema teórico, de conformidad con lo explicado en la nota anterior. 
El cálculo, tal y como esa actividad quedó descrita en sección anterior, consiste en el manejo de definiciones formales bien logradas. La reflexión, en contraste con el cálculo, implica más bien un aventurarse más allá de las definiciones logradas, en busca de nuevos contextos. ${ }^{10}$ Pero la reflexión es impulsada a esos nuevos contextos precisamente al transformar las definiciones vigentes en contradicciones irritantes. Mientras advienen las nuevas definiciones, exigidas por el aventurarse de la reflexión y contingentes al cambio de contexto, la contradicción aflora en las viejas definiciones como dramático testimonio de la necesidad imperiosa de redefinir. Allí puede permanecer por un período considerable, pues el proceso de redefinición no es un proceso fácil. No se trata de redefinición de términos (dentro de un contexto fijo), sino de verdadera redefinición contextual: el cambio de contexto será el que modifique el sentido de los términos. No se trata de que la definición se logre por sustitución de unos términos por otros en el definiens; eso, por hipótesis, no es posible en las situaciones críticas en que surgen contradicciones. Cambiamos la definición mayormente mediante variación de la significación contextual de los términos usados en el definiens, sean éstos los mismos antes empleados $\mathrm{u}$ otros distintos. $\mathrm{Y}$ esta variación de significación contextual debe ser de tal naturaleza que là modificación del sentido de los términos conflictivos se haga sin afectar adversamente el funcionamiento de multitud de esencias o explicaciones causales eficientes y no conflictivas del resto del paradigma. Por eso, por la inherente dificultadad de la redefinición contextual, querer resolver rápidamente una contradicción es frívolo y anticientífico. El científico de valor, como el hombre de valor, sabe esperar y vivir en contradicciones. No porque le guste la contradicción o la considere un valor positivo en sí misma; sino porque está convencido de la seriedad de la em-

\footnotetext{
10 Podríamos decir que la reflexión corresponde eminentemente a la filosofía, en tanto que el cálculo a la ciencia. Pero habría que proveer para períodos o zonas filosóficas en la ciencia y períodos o zonas científicas en la filosofía.
} 
presa de modificar una esencia (la "seriedad del concepto" de que hablaba Hegel). ${ }^{\text {d1 }}$ Por su convencimiento profundo de que muchas veces conviene agudizar las contradicciones para que su poder penetrante sirva para revelar con mayor claridad la esencia de las cosas y de las situaciones.

\section{Conclusión}

He planteado una contradicción entre un concepto lógico o analítico de la contradicción, con connotaciones desfavorables como "vicio del razonamiento", y un concepto dialéctico o sintético, con connotaciones favorables como "cifra o signo de contenido esencial". A través de una reflexión contextual sobre el tema, he llegado a la conclusión de que estos dos conceptos forman una contradicción en el sentido dialéctico, que sólo puede removerse o superarse mediante una reelaboración del contexto teórico respectivo $;^{\mathbf{1 2}}$ creo haber contribuido a esa reelaboración con el mismo proceso que me llevó a esta conclusión. En el nuevo contexto, dicha contradicción dialéctica queda redefinida como una contradicción analítica que clama por su remoción mediante reelaboración de su contexto. Nótese que la reelaboración de contexto que resuelve la contradicción que da origen a este trabajo contiene un principio eminentemente general, de suyo aplicable a la interpretación de cualquier contradioción dialéctica. De ahí que me atreva a postular las siguientes dos tesis:

1) La existencia de una contradicción (dialéctica) entre los dos conceptos de contradicción examinados revela una inadecuación del contexto teórico en que ella surge (presumiblemente algún tipo de filosofía de inclinación positivista) y reclama una reelaboración de este contexto.

11 G. W. F. Hegel, Fenomenología del Espíritu, Fondo de Cultura Económica, México, D. F., 1966, p. 9. "Der Ernst des Begriffs" es una de las frases famosas del filósofo dialéctico por excelencia.

12 Cfr. Wilhelm Dilthey, Teoria de la concepción del mundo, Fondo de Cultura Económica, México, D. F., 1945, p. 8: "No se puede resolver una antinomía sobre el suelo mismo en que ha nacido." 
2) La reelaboración que es necesaria para remover esa inadecuación comprende una generalización del contenido de la primera tesis de modo que se entienda aplicable a cualquier contradicción de parecida naturaleza. 


\section{SUMMARY}

There are two ways of considering contradiction in ordinary language and philosophical writings: the logical or analytical concept, which considers it a defect of reasoning; and the dialectical or synthetic conception which considers it as a symptom of ontological density. The opposition between both concepts is at once an example of contradiction (of a dialectical kind). This situation is examined in order to decide if the two uses of the term "contradictory" are equivocal, univocal, or analogous: the dialectical use seems to contain all the meaning of the analytical use as well as an amount of additional meaning.

The object of knowledge is the concrete or real. But in order to reach the concrete it is necessary to pass through the abstract. The synthesis, which constitutes our orientation in the world, starts out as an analysis consisting in separating things that are known into elements or factors. Analysis and synthesis are related in the same way as form to content, text to context. An analysis which separates text from context, takes away part of its content; in the end, a form will remain, a text with a minimum of context. But the synthesis should reintegrate the text into the context; then the concrete will become illuminated by its passage through the abstract.

Content and formalism correspond to two fundamental linguistic dimensions: semantics and syntax. The syntactical is the minimum, immediate and manageable context. The semantical is the expansive context, which takes you from itself into ever wider circles of meaning. The syntactical is a matter of calculation; the semantical, of reflection. All thought is at one and the same time both syntactical and semantical. A thought consisting of pure reflection would be incommunicable and impractical; it would continue to float indefinitely in an endless indifference without ever deciding what it wants to say or do. On the other hand, absolute formalism is impossible, because to formalize is to substitute one content for another, which for a given purpose is considered to be simpler. All formalization consits in the constitution of a model, and this model also has content. The pure structure, without a material substratum to incarnate it, simply does not exist.

To know is to assimilate essences. The empiricist ideal does not include that we should dispense with the use of the term "es- 
sence". It is sufficient that we cast off the notion that there are eternal essences and understand that the essence is dependent upon the context. What is not essential in one context becomes so in another, and vice versa. Nor is the concept "essence" independent from a given context, and it should now be given a definition of "essence" different from the one given in the days of Aristotle. But the term "essence" is indispensable; if objects admit of classification, if uniformity exists in nature, we must have essences so as to be able to talk about it. The universe is the interrelationship of events; every object takes us to other.objects, to such a degree that we can conceive of the universe as an immense network of interrelationships. In this network the especially dense nodes constitute essences. Understanding supposes that we are able to separate the essential from the accidental, the strong connexions from the weak ones. But there is no distinction made in nature between the strong and the week connections. Essence is not only contextual, but also admits degrees.

Everything real is interconnection; knowledge is real, it is therefore interconnection. Knowledge has as its object the real, therefore it is a triple interconnection. In order to know we interact with an interaction. It is never given in experience the pair, subjectobject, but the triple, subject-object-context. All interaction is material, i.e. is a work. Knowledge has as its proper work analysis, which tries to force the context out of its triple connection in order to, methodically, attempt a double relationship between mind and object. The eliminated context reacts by infiltrating itself into the formal structure as an insufficiency which is very often a contradiction. The contradiction may serve as a key to redefinition, as a testimony of the need to restore or amplify a context. In this sense, and only in this sense, contradiction is a sympton of the complexity of the real, it shows the essence. Finding contradictions can signify the discovery of essences, since the contradiction makes necessary an amplification of the context which enriches the content of the former definitions. There may be contradictions that are more or less severe, just as there are richer and less rich essences. It is possible to have degrees of contradiction, just as there may be degrees of essence. The principal contradiction, which demands the most severe context reformulations, is the essence of the situation in the most proper sense. Thought progresses from definition to definition through contradiction: this is what constitutes dialectics. Contradiction ceases to be the absurd if it is understood as a call upon language to reformulate the universe of discourse. 
Calculus consists in dealing with definitions. Reflection, by contrast, implies venturing beyond the definitions obtained, in search of more extensive contents. Reflection is prompted toward these new contents by the transformation of the presently accepted definitions into irritating contradictions. The contradiction crops out in the definition as an imperious need for redefinition. It can remain there for a considerable time, since the process of contextual redefinition is not an easy one. Because of the inherent difficulty of contextual redefinition, pretending to solve a contradiction quickly is frivolous and antiscientific. The worthy scientist, just as the worthy man, knows how to wait and live among contradictions, because of his conviction that it is often a good thing for contradictions to subsist and even become sharper in order that his powers of penetration may reveals with greater clarity the essence of the situation.

In conclusion, it is established that both concepts of contradiction, when they themselves form a contradiction (a dialectical one), reveal an inadequacy in the theoretic context and call for a reelaboration of that context.

The reelaboration required for removing the inadequacy includes a generalization of the thesis in such a way that it may be understood to apply to any contradiction of a similar nature: every dialectic contradiction is an analytic contradiction which calls for removal through the reelaboration of its context. 